\title{
Property C for ODE and Applications to an Inverse Problem for a Heat Equation
}

by

\author{
A. G. RAMM \\ Presented by Bogdan BOJARSKI
}

Summary. Let $\ell_{j}:=-d^{2} / d x^{2}+k^{2} q_{j}(x), k=$ const $>0, j=1,2,0<\operatorname{essinf} q_{j}(x) \leq$ $\operatorname{ess} \sup q_{j}(x)<\infty$. Suppose that $(*) \int_{0}^{1} p(x) u_{1}(x, k) u_{2}(x, k) d x=0$ for all $k>0$, where $p$ is an arbitrary fixed bounded piecewise-analytic function on $[0,1]$, which changes sign finitely many times, and $u_{j}$ solves the problem $\ell_{j} u_{j}=0,0 \leq x \leq 1, u_{j}^{\prime}(0, k)=0, u_{j}(0, k)=1$. It is proved that $(*)$ implies $p=0$. This result is applied to an inverse problem for a heat equation.

1. Introduction. Property $\mathrm{C}$ stands for completeness of the set of products of solutions to homogeneous equations. This notion was introduced by the author in [3], [4, [8], and used widely as a powerful tool for proving uniqueness theorems for many inverse problems ([5]-[12]). In [8] Property C was proved for the pair of operators

$$
\left\{\frac{d^{2}}{d x^{2}}+k^{2}-q_{1}(x), \frac{d^{2}}{d x^{2}}+k^{2}-q_{2}(x)\right\},
$$

where

$$
q_{1}, q_{2} \in L_{1,1}:=\left\{q: q=\bar{q}, \int_{0}^{\infty}(1+x)|q(x)| d x<\infty\right\} .
$$

The novel point in our paper is the proof of Property $\mathrm{C}$ for a pair of differential operators with a different dependence on the spectral parameter. This new version of Property $\mathrm{C}$ turns out to be basic, for example, in the proof of uniqueness for an inverse problem for a heat equation with a discontinuous thermal conductivity.

2010 Mathematics Subject Classification: 35R30, 74J25, 34E05.

Key words and phrases: property C, inverse problems, heat equation, discontinuous coefficients. 
The aim of this paper is to prove Property $\mathrm{C}$ for the pair $\left\{\ell_{1}, \ell_{2}\right\}$, where

$$
\begin{gathered}
\ell_{j}:=-\frac{d^{2}}{d x^{2}}+k^{2} q_{j}(x), \quad j=1,2, \\
0<\operatorname{essinf} q_{j}(x) \leq \operatorname{ess} \sup q_{j}(x)<\infty,
\end{gathered}
$$

and $k$ is a positive number. These standing assumptions about $q_{j}$ are not repeated in the formulation of the theorems below.

Denote by $M_{0}$ the set of $L^{1}(0,1)$ functions which change sign at most finitely many times in the interval $[0,1]$.

We say that a function $p$ has finitely many changes of sign in $[0,1]$ if there is a partition of this interval into finitely many subintervals on which the function does not change sign, that is, $p$ is either non-negative or non-positive on each of the subintervals of this partition.

DeFINITION 1. Let

$$
\ell_{j} u_{j}(x, k)=0, \quad u_{j}^{\prime}(0, k)=0, \quad u_{j}(0, k)=1, \quad 0 \leq x \leq 1,
$$

and let $W \subset L^{1}(0,1)$ be some set. If, for all $h \in W$, the orthogonality relation

$$
\int_{0}^{1} h(x) u_{1}(x, k) u_{2}(x, k) d x=0 \quad \forall k>0,
$$

implies $h=0$, then we say that the pair $\left\{\ell_{1}, \ell_{2}\right\}$ has Property $C$ for the set $W$.

Our first result is:

Theorem 1. The pair $\left\{\ell_{1}, \ell_{2}\right\}$ has Property $C$ for the set $M_{0}$.

Remark. The set $M_{0}$ is dense in $L^{2}(0,1)$. Therefore one might expect that the result of Theorem 1 implies that the pair $\left\{\ell_{1}, \ell_{2}\right\}$ has Property $\mathrm{C}$ in $L^{2}(0,1)$. However, such a conclusion does not follow from Theorem 1: one can construct an example of a set $W$, dense in $L^{2}(0,1)$, and a set $\left\{\phi_{j}\right\}_{j=1}^{\infty}$, such that the conditions $f \in W$ and $\left(f, \phi_{j}\right)=0$ for all $j$ imply $f=0$, but there exists an $h \in L^{2}(0,1), h \neq 0$, such that $\left(h, \phi_{j}\right)=0$ for all $j$. See an example in [13, pp. 164-165]. Here is another example. Let $W=C(0,1)$. Then $W$ is dense in $L^{2}(0,1)$. Choose $h \notin W, h \in L^{2}(0,1), h \neq 0$. Let $V$ be the orthogonal complement to $h$ in $L^{2}(0,1)$, and $\left\{\phi_{j}\right\}_{j=1}^{\infty}$ be an orthonormal basis of $V$. If $f \in C(0,1)$ and $\left(f, \phi_{j}\right)=0$ for all $j$, then $f=c h$, where $c=$ const. Since $f \in C(0,1)$ on the one hand, and $f=c h \notin C(0,1)$, unless $c=0$, on the other hand, we conclude that $c=0$ and $f=0$. However, $h \neq 0$ and $\left(h, \phi_{j}\right)=0$ for all $j$. Therefore the set $\left\{\phi_{j}\right\}_{j=1}^{\infty}$ is not complete in $L^{2}(0,1)$, but there is no $f \in C(0,1), f \neq 0$, such that $\left(f, \phi_{j}\right)=0$ for all $j$.

It is an open problem whether the pair $\left\{\ell_{1}, \ell_{2}\right\}$ has Property $\mathrm{C}$ in $L^{2}(0,1)$. Let us give an example of applications of Property C. 
Denote by $M$ a set of real-valued integrable functions such that if $q_{j} \in M$, $j=1,2$, are arbitrary members of $M$, then the function $p(x):=q_{2}(x)-q_{1}(x)$ is in $M_{0}$.

Let us mention some examples of such sets $M$ :

1) $M_{1}$ is the set of piecewise-constant functions on $[0,1]$ with finitely many discontinuity points (see [2]),

2) $M_{2}$ is the set of piecewise-analytic real-valued functions on $[0,1]$ with finitely many discontinuity points,

3) $M_{3}$ is the set of functions of the form $q_{0}+q$, where $q_{0}$ is a fixed real-valued integrable function, and $q \in M_{2}$.

Consider the problem

$$
\begin{gathered}
U_{t}=\left(a(x) U^{\prime}\right)^{\prime}, \quad 0 \leq x \leq 1, \quad t>0 ; \quad U^{\prime}:=\frac{\partial U}{\partial x}, \\
U(x, 0)=0, \quad U(0, t)=0, \quad U(1, t)=F(t), \\
a(1) U^{\prime}(1, t)=G(t) .
\end{gathered}
$$

Denote by $M_{2}^{\prime}$ the subset of functions in $M_{2}$ which satisfy the inequalities

$$
0<\inf a(x) \leq \sup a(x)<\infty .
$$

The function $F$ satisfies $F \not \equiv 0, F \geq 0, F(t)=0$ if $t>T$, where $T>0$ is an arbitrary fixed number, and $F \in L^{1}([0, T])$.

Problem (3)-(4) has a unique solution.

The above assumptions about $a$ and $F$ will not be repeated in the formulation of Theorem 2.

The function $G$ is an (extra) measured datum, which is the heat flux at the point $x=1$.

The extra datum $G$ cannot be given arbitrarily: it is determined by $F$ since problem (3)-(4) is uniquely solvable. If $G$ is an arbitrary function, no matter how smooth it is, problem (3)-(5) may have no solutions.

In this paper we are concerned with the uniqueness of solution to the inverse problem, stated below, and do not discuss the problem of existence of solutions, that is, conditions on the data $F$ and $G$ under which the data are compatible.

The inverse problem is:

$\mathrm{IP}_{1}$ : Given $\{F(t), G(t)\}_{t>0}$, find $a \in M_{2}^{\prime}$.

The function $a \in M_{2}^{\prime}$ has finitely many discontinuity points, and the solution to (3)-(4) is understood in the weak sense. 
Let us formulate $\mathrm{IP}_{1}$ in an equivalent but different form. Let

$$
\begin{aligned}
v(x, \lambda) & :=\int_{0}^{\infty} U(x, t) e^{-\lambda t} d t, \quad f(\lambda):=\int_{0}^{\infty} F(t) e^{-\lambda t} d t, \\
g(\lambda) & :=\int_{0}^{\infty} G(t) e^{-\lambda t} d t .
\end{aligned}
$$

Take the Laplace transform of (3)-(5) to get

$$
\begin{gathered}
-\left(a(x) v^{\prime}\right)^{\prime}+\lambda v=0, \quad 0 \leq x \leq 1, \quad \lambda>0, \\
v(0, \lambda)=0, \quad v(1, \lambda)=f(\lambda), \quad a(1) v^{\prime}(1, \lambda)=g(\lambda) .
\end{gathered}
$$

Let $u(x, \lambda):=a(x) v^{\prime}(x, \lambda)$. Differentiate (8) to get

$$
\begin{gathered}
-u^{\prime \prime}+\lambda a^{-1}(x) u=0, \quad 0 \leq x \leq 1, \quad u^{\prime}(0, \lambda)=0, \\
u(1, \lambda)=g(\lambda), \quad u^{\prime}(1, \lambda)=\lambda f(\lambda) .
\end{gathered}
$$

Let

$$
\lambda=k^{2}, \quad k>0 ; \quad a^{-1}(x):=q(x) .
$$

Then $q(x) \geq c_{0}>0, c_{0}=$ const,

$$
-u^{\prime \prime}+k^{2} q(x) u=0, \quad 0 \leq x \leq 1, \quad u^{\prime}\left(0, k^{2}\right)=0, \quad u\left(1, k^{2}\right)=g\left(k^{2}\right),
$$

and the data are $\left\{g\left(k^{2}\right), k^{2} f\left(k^{2}\right)\right\}_{k>0}$.

Note that if $a \in M_{2}^{\prime}$, then $q \in M_{2}^{\prime}$.

Now, $\mathrm{IP}_{1}$ can be reformulated as follows:

$\mathrm{IP}_{2}$ : Given the data $\left\{g\left(k^{2}\right), k^{2} f\left(k^{2}\right)\right\}_{k>0}$, find $q \in M_{2}^{\prime}$.

Our second result is:

ThEOREM 2. IP 2 has at most one solution.

Theorem 2 implies the uniqueness of solution to $\mathrm{IP}_{1}$ in the class of piecewise-analytic strictly positive functions $a$ with finitely many discontinuity points in $[0,1]$.

In the literature, $\mathrm{IP}_{1}$ has been considered earlier (see, e.g., 9], [10] and references therein) in the case when $a \in H^{2}([0,1])$, where $H^{2}$ is the Sobolev space. For piecewise-constant thermal conductivity coefficients $a(x)$ with finitely many discontinuity points, $\mathrm{IP}_{1}$ was studied recently in [2]. An inverse problem for equation (3) with different extra data, namely $U\left(\xi_{n}, t\right)$ for $t>0$, $\xi_{n} \in[0,1], 1 \leq n \leq N, \min _{1 \leq n \leq N}\left|\xi_{n}-\xi_{n+1}\right| \geq \sigma>0$, where $\sigma$ is a fixed number, $N=3 \nu$, and $\nu$ is the number of discontinuity points of $a$, was studied in [1].

In $\mathrm{IP}_{1}$ the extra data are collected at just one point $x=1$.

Our arguments prove the uniqueness result of Theorem 2 in the case when the data in $\mathrm{IP}_{1}$ are the values $\{F(t), G(t)\}_{t \in[0, T+\epsilon]}$ for an arbitrarily small $\epsilon>0$. These data determine $a$ uniquely because the solution $U(x, t)$ 
is an analytic function of $t$ in a neighborhood of the set $(T, \infty)$, so the knowledge of $U(x, t)$ on $[0, T+\epsilon]$ determines $U(x, t)$ uniquely for all $t>0$.

\section{Proofs}

Proof of Theorem 1. The solution to (1) solves the equation

$$
u_{j}(x, k)=1+k^{2} \int_{0}^{x}(x-s) q_{j}(s) u_{j}(s, k) d s, \quad x \geq 0, \quad j=1,2 .
$$

This is a Volterra equation. It has a unique solution $u_{j}(x, k)$. This solution has the following properties:

$$
\begin{gathered}
u_{j}(x, k) \geq 1, \quad u_{j}^{\prime}(x, k) \geq 0, \quad u_{j}^{\prime \prime}(x, k)>0, \quad 0 \leq x \leq 1, \\
\frac{\partial^{i} u_{j}}{\left(\partial k^{2}\right)^{i}} \geq 0, \quad i=1,2, \ldots, \\
\lim _{k \rightarrow \infty} \frac{u_{j}(y, k)}{u_{j}(x, k)}=0, \quad 0 \leq y<x \leq 1 .
\end{gathered}
$$

Properties (14)- (15) are immediate consequences of (13). Let us prove 116$)$. One has

$$
u_{j}(x, k)=u_{j}(y, k)+\int_{y}^{x} u_{j}^{\prime}(s, k) d s .
$$

From (13) and (14) one obtains

$$
u_{j}^{\prime}(x, k)=k^{2} \int_{0}^{x} q_{j}(s) u_{j}(s, k) d s \geq k^{2} \int_{0}^{x} q_{j}(s) d s .
$$

From (17) and (18) one gets

$$
\begin{aligned}
\frac{u_{j}(x, k)}{u_{j}(y, k)} & =1+\int_{y}^{x} \frac{u_{j}^{\prime}(s, k)}{u_{j}(y, k)} d s=1+k^{2} \int_{y}^{x} \frac{\int_{0}^{s} q_{j}(z) u_{j}(z, k) d z}{u_{j}(y, k)} d s \\
& \geq 1+k^{2} \int_{y}^{x} d s \int_{y}^{s} q_{j}(z) d s \geq 1+\frac{1}{2} k^{2} c_{0}(x-y)^{2} \rightarrow \infty
\end{aligned}
$$

as $k \rightarrow \infty$, where $q_{j}(x) \geq c_{0}=$ const $>0$.

Thus, (16) is proved.

Since $h \in M_{0}$, the interval $[0,1]$ is a union of finitely many intervals without common interior points on each of which the function $h$ keeps constant sign. Let $[z, 1]$ be such an interval. We want to prove that $h=0$ on this interval. If this is done then similarly, in a finite number of steps, one proves that $h=0$ on the whole interval $[0,1]$, and thus the proof of Theorem 1 is complete. 
Let us rewrite relation (2) as

$$
\begin{aligned}
\int_{z}^{1} h(x) u_{1}(x, k) u_{2}(x, k) d x & =-\int_{0}^{z} h(x) u_{1}(x, k) u_{2}(x, k) d x \\
& \leq u_{1}(z, k) u_{2}(z, k) \int_{0}^{z}|h(x)| d x,
\end{aligned}
$$

where the monotonicity and positivity of $u_{j}$ were used (see (14)). Without loss of generality assume that $h(x)>0$ on $[z, 1]$ and fix an arbitrary $y \in$ $(z, 1)$. Then

$$
\int_{z}^{1} h(x) u_{1}(x, k) u_{2}(x, k) d x \geq \int_{y}^{1} h(x) d x u_{1}(y, k) u_{2}(y, k) .
$$

From $(20)$ and $(21)$ one gets

$$
\int_{y}^{1} h(x) d x \leq \frac{u_{1}(z, k) u_{2}(z, k)}{u_{1}(y, k) u_{2}(y, k)} \int_{0}^{z}|h(x)| d x, \quad y>z .
$$

Let $k \rightarrow \infty$ in 22 and use 16 to get $\int_{y}^{1} h(x) d x=0$. Since $h(x) \geq 0$ on $[z, 1]$, it follows that $h=0$ on $[y, 1]$. Since the point $y \in(z, 1)$ is arbitrary, it follows that $h=0$ on $[z, 1]$. Theorem 1 is proved.

Proof of Theorem 2. Assume there are pairs of functions $\left\{\psi_{1}, q_{1}\right\}$ and $\left\{\psi_{2}, q_{2}\right\}$ which solve (12) and (11) with $\lambda=k^{2}$. Let $w=\psi_{1}-\psi_{2}$. Then

$$
w^{\prime}(0, k)=w(1, k)=w^{\prime}(1, k)=0,
$$

and

$$
-w^{\prime \prime}+k^{2} q_{1}(x) w=k^{2} p(x) \psi_{2}, \quad p(x):=q_{2}(x)-q_{1}(x) .
$$

Multiply (24) by $u_{1}(x, k)$, integrate over $[0,1]$, and then integrate by parts using (23) to obtain

$$
k^{2} \int_{0}^{1} p(x) u_{1}(x, k) \psi_{2}(x, k) d x=0 \quad \forall k>0 .
$$

Since $\psi_{2}(x)=c(k) u_{2}(x, k)$, where $c(k)=$ const $\neq 0$, and $p \in M_{0}$, it follows from (25) and Theorem 1 that $p=0$, so $q_{1}=q_{2}$.

Theorem 2 is proved.

\section{References}

[1] S. Gutman and J. Ha, Identifiability of piecewise constant conductivity in a heat conduction process, SIAM J. Control Optim. 46 (2007), 694-713. 
[2] N. S. Hoang and A. G. Ramm, An inverse problem for a heat equation with piecewiseconstant thermal conductivity, J. Math. Phys. 50 (2009), 063512.

[3] A. G. Ramm, On completeness of the products of harmonic functions, Proc. Amer. Math. Soc. 99 (1986), 253-256.

[4] -, Completeness of the products of solutions to PDE and uniqueness theorems in inverse scattering, Inverse Problems 3 (1987), L77-L82.

[5] -, Multidimensional inverse problems and completeness of the products of solutions to PDE, J. Math. Anal. Appl. 134 (1988), 211-253; Addenda, ibid. 139 (1989), 302.

[6] - Completeness of the products of solutions of PDE and inverse problems, Inverse Problems 6 (1990), 643-664.

[7] —, Necessary and sufficient condition for a PDE to have property $C$, J. Math. Anal. Appl. 156 (1991), 505-509.

[8] - Property C for ODE and applications to inverse problems, in: Operator Theory and Its Applications, Amer. Math. Soc., Fields Inst. Comm. 25, Providence, RI, Amer. Math. Soc., 2000, 15-75.

[9] —, An inverse problem for the heat equation, J. Math. Anal. Appl. 264 (2001), 691-697.

[10] -, An inverse problem for the heat equation II, Appl. Anal. 81 (2002), 929-937.

[11] -, One-dimensional inverse scattering and spectral problems, Cubo Math. J. 6 (2004), 313-426.

[12] - Inverse Problems, Springer, New York, 2005.

[13] —, Scattering by Obstacles, Reidel, Dordrecht, 1986.

A. G. Ramm

Department of Mathematics

Kansas State University

Manhattan, KS 66506-2602, U.S.A.

E-mail: ramm@math.ksu.edu

Received May 12, 2009;

received in final form November 4, 2009 